\title{
The Role of Amnesia Caused by Cerebral Hypoperfusion on Depression-Like Behavior in Male Wistar Rats
}

\author{
Ashkan Divanbeigi ${ }^{1,2}$, Mohammad Nasehi ${ }^{*}$, Sepideh Amiri ${ }^{4}$, Mohammad Reza Zarrindast $^{1,3,5}$ \\ ${ }^{1}$ Institute for Cognitive Science Studies (ICSS), Tehran, Iran \\ ${ }^{2}$ Functional Neurosurgery Research Center, Shahid Beheshti University of Medical Sciences, Tehran, Iran \\ ${ }^{3}$ Cognitive and Neuroscience Research Center (CNRC), Tehran Medical Branch, Islamic Azad University, Tehran, Iran \\ ${ }^{4}$ Shefa Neuroscience Research Center, Khatam Alanbia Hospital, Tehran, Iran \\ ${ }^{5}$ Center for Addiction Studies, Tehran University of Medical Sciences, Tehran, Iran
}

\section{Article Info:}

\section{ABSTRACT}

Introduction: Anxiety disorders, depression, and dementia are common in elderly, which may overlap in various neurological disorders. Stress can be a risk factor for long-term depression. It also suggests that depression plays a role in developing dementia. Amnesia is the most problem in dementia. Is amnesia a risk factor for depression? Considering the common underlying mechanisms for causing these disorders, the purpose of this study is to investigate the role of amnesia in developing depression. Materials and Methods: Fortyeight wistar rats were divided into two main groups (sham-A and intervention-B) and four subgroups (Control-C, Chronic mild stress-D, hypoperfusion without stress-E, hypoperfusion with Mild stress-F). Permanent occlusion of bilateral common carotid artery was performed to induce cerebral hypoperfusion. Radial arm maze test and chronic unpredictable mild stress were used to evaluate amnesia and depression, respectively. Forced swim test was performed to assess severity of depression and finally we used neuronal count for evaluating cellular degeneration in the CA1 hippocampus region. Results: Cerebral hypoperfusion causes amnesia and significantly reduced the mean number of cells in the hippocampal CA1 area. Cerebral hypoperfusion and stress significantly increased the incidence of depression-like behavior. Conclusion: Amnesia caused by cerebral hypoperfusion can increase the risk of depression, especially under stressful situations.

\section{Key words:}

1. Amnesia

2. Dementia

3. Brain Ischemia

4. Depression

*Corresponding Author: Mohammad Nasehi

E-mail: Nasehi@iricss.org 
نقش فراموشى ناشى از هييويرفيوزن مغزى در رفتارهاى مشابه افسردَى در موشهاى صحر ايیى نر نزاد ويستار

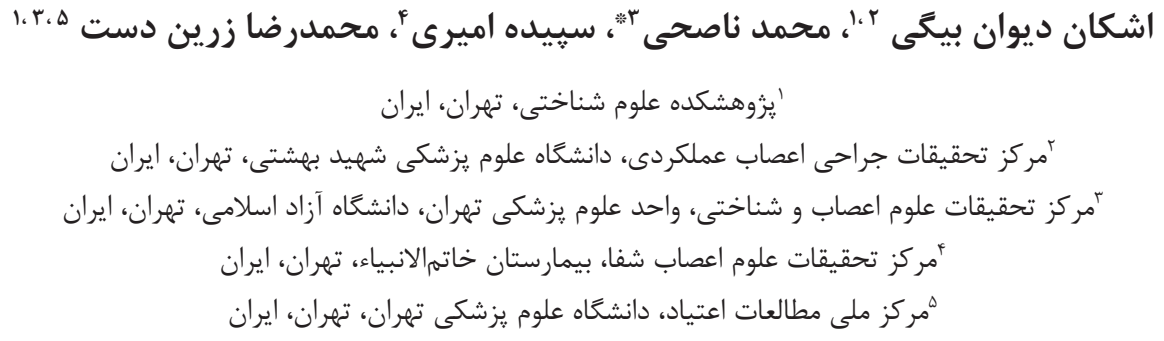

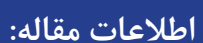

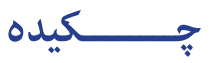

مقدمه: اختلالات اضطرابى، افسردىى و دمانس در سنين بالا شايع است كه ممكن است در اختلالالات

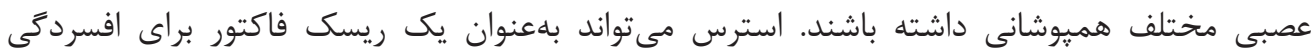

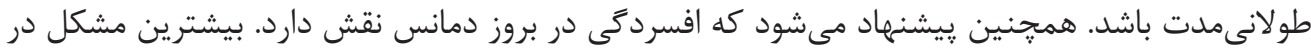

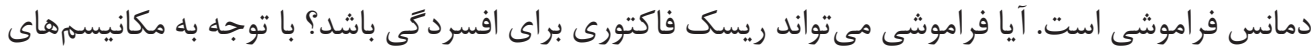

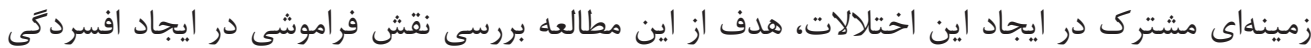

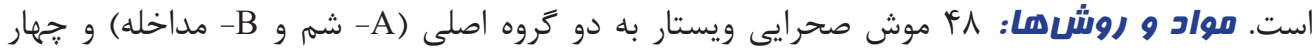

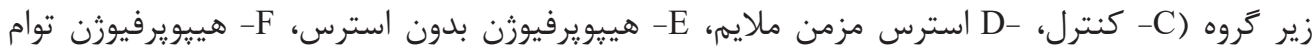

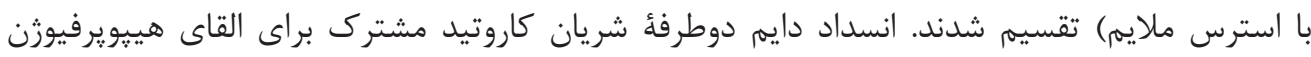

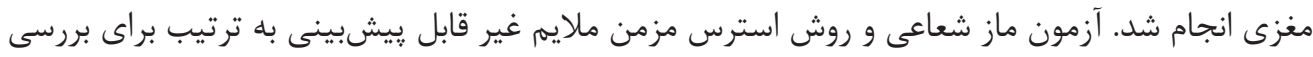

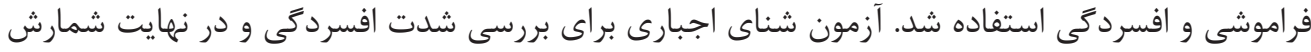

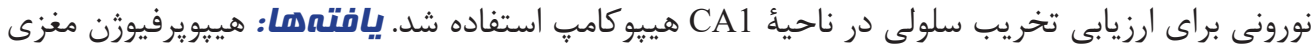

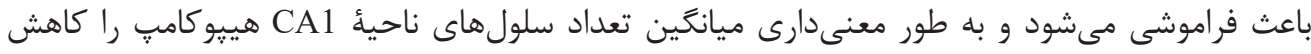

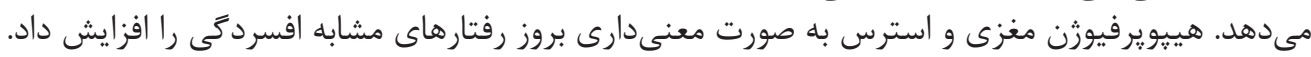

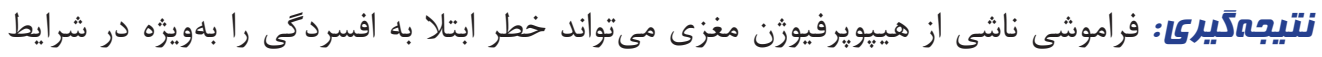

: كليد وازهها:

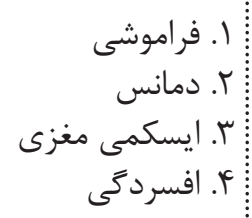




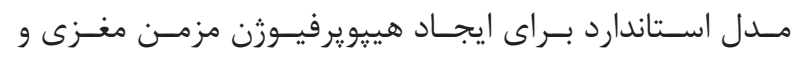

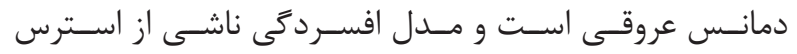

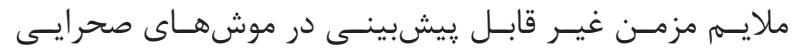

اسـتفاده كرديـهم (11 ، ـ • (1).

مواد و روشها

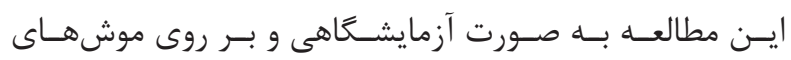

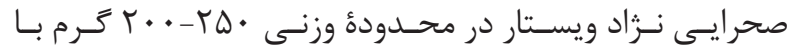

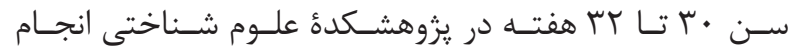

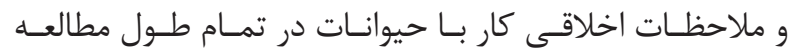

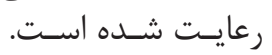

$\log$

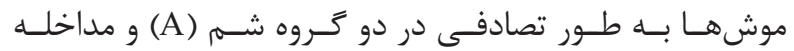

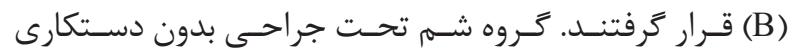

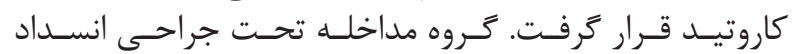

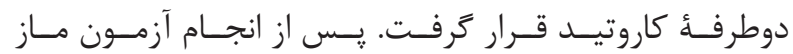

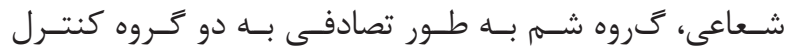

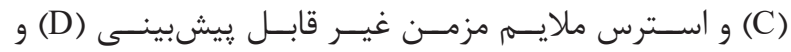

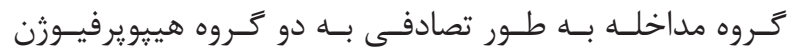

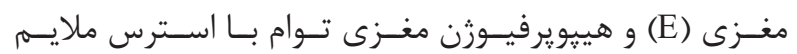

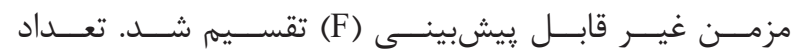

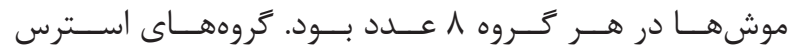

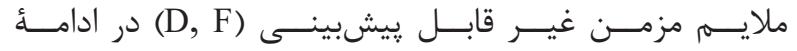

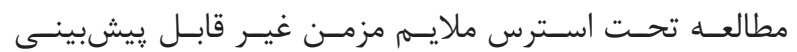
قـرار كرفتنـد. تحست

روش جر احى و آمادهسازى

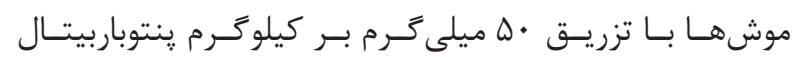

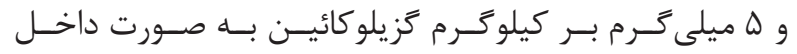

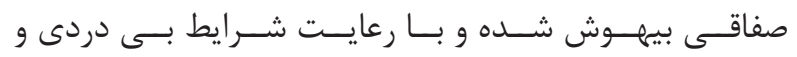

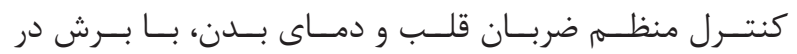

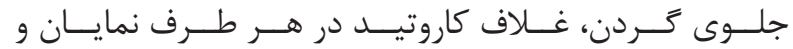

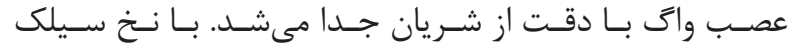

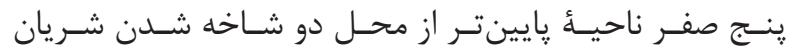

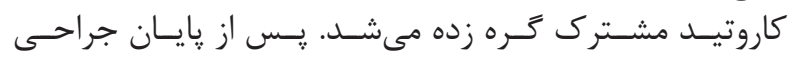

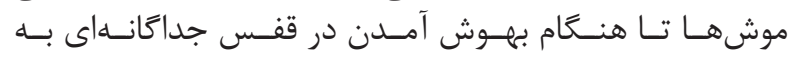

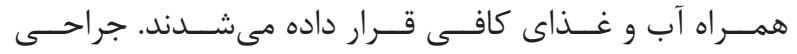

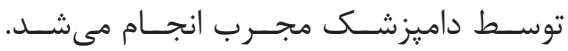

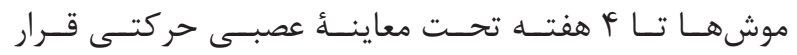

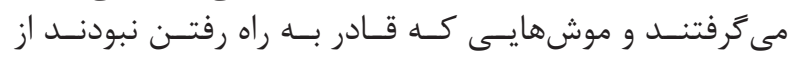

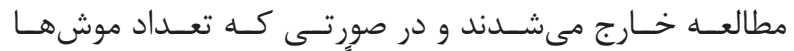

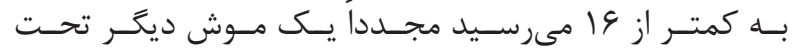

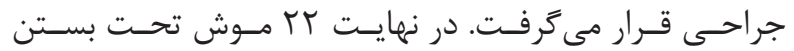

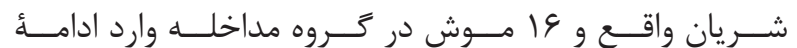

مطالعــه شـــند.
مقدمه

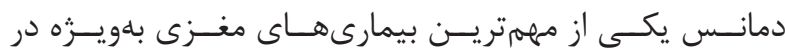

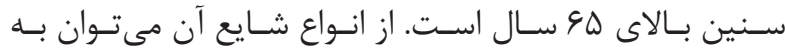

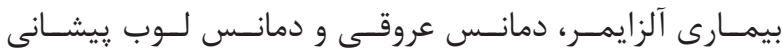

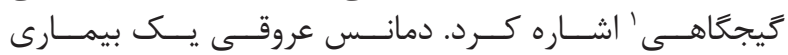

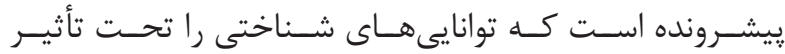

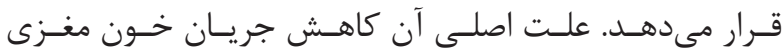

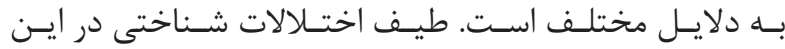

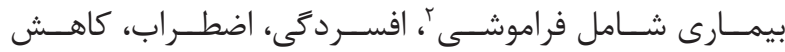

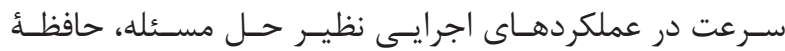

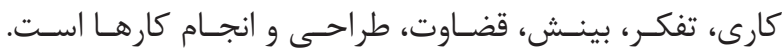

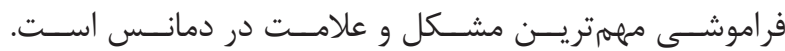

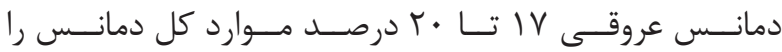

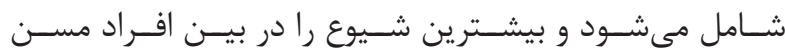

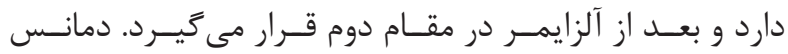

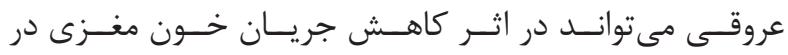

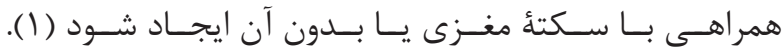

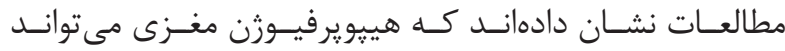

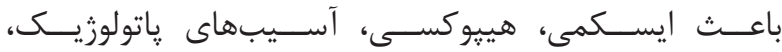

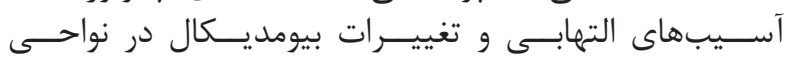

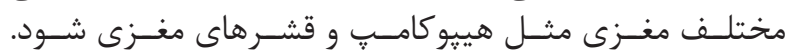

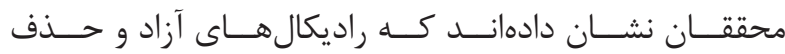

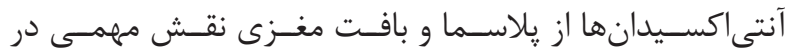

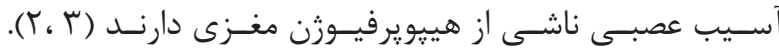

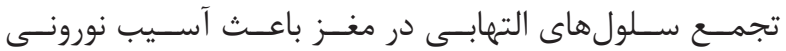

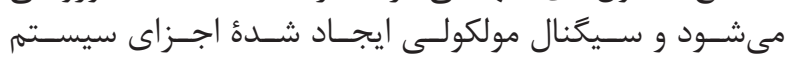

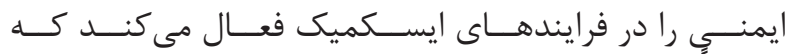

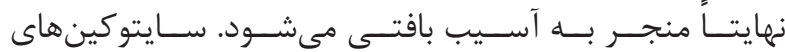

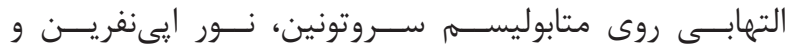

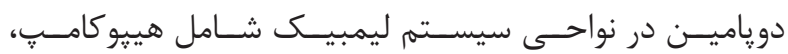

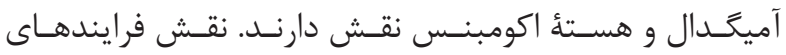

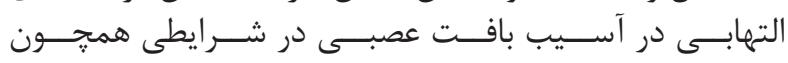

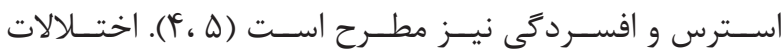
اضطرابــى، افســرد

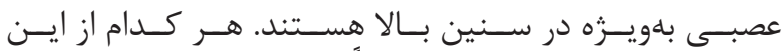

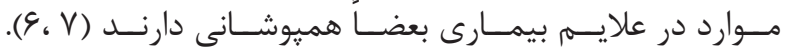

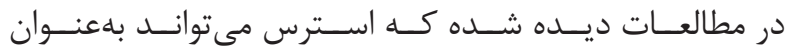

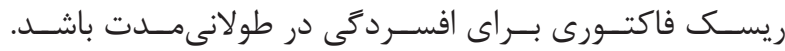

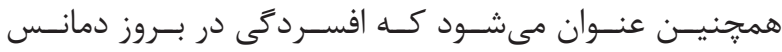

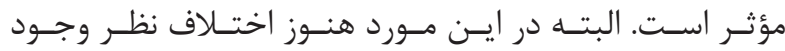

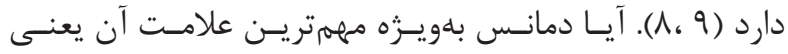

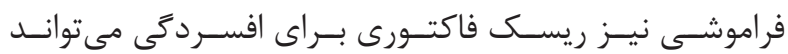

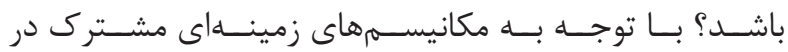

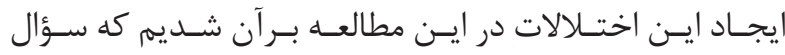

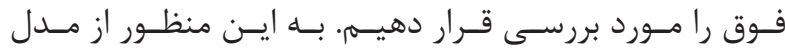

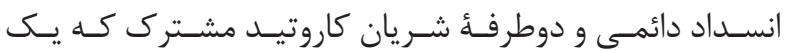

${ }^{1}$ Frontotemporal lobe

${ }^{2}$ Amnesia 


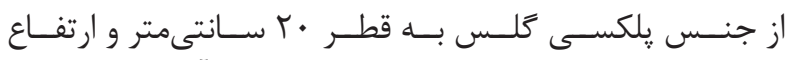

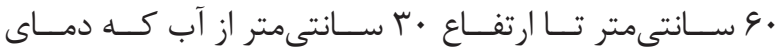

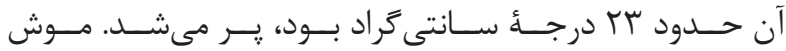

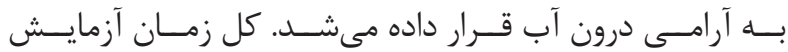

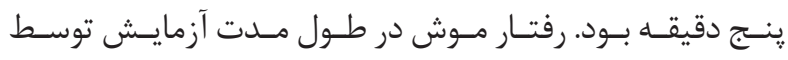

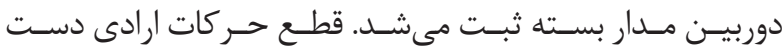

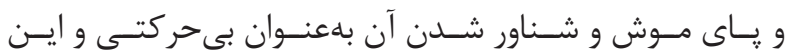

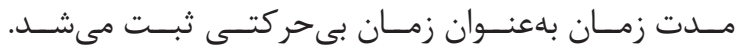

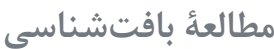

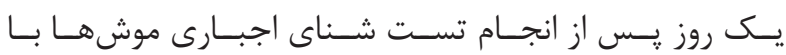

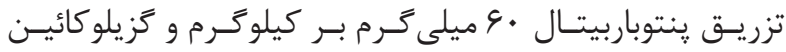

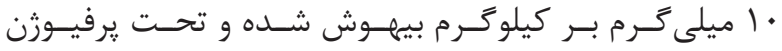

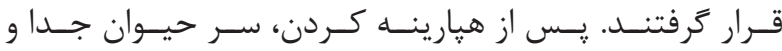

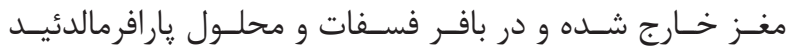

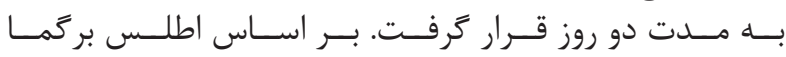

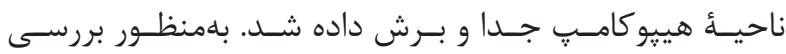

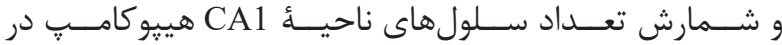

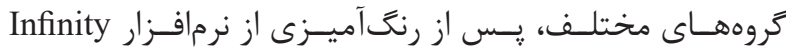

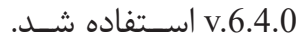

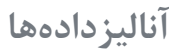

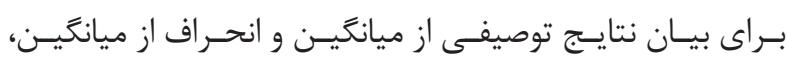

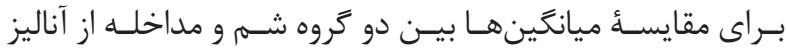

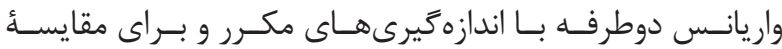

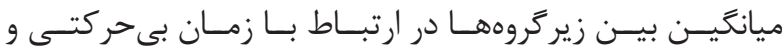

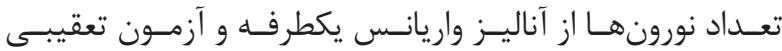

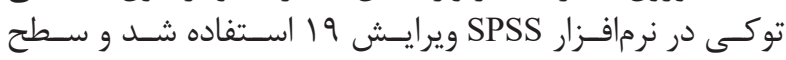

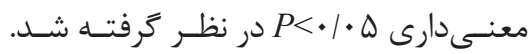

بافته ها

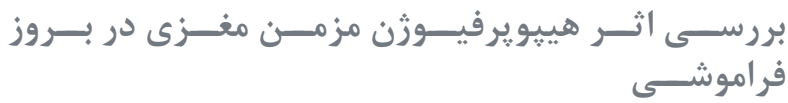
حافظهُ كارى

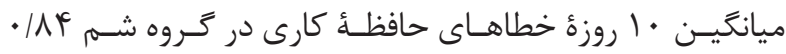
مأl

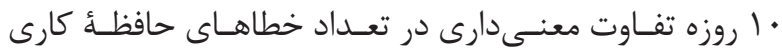

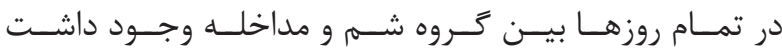

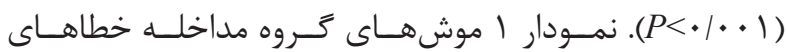

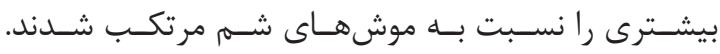

حافظةُ مر جع

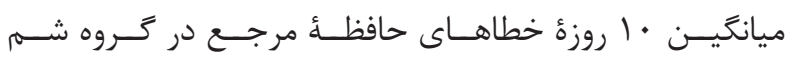
س م/ •

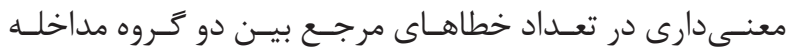

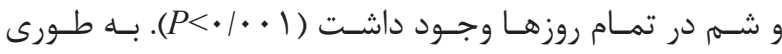

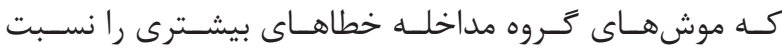

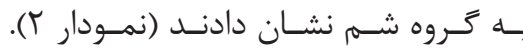

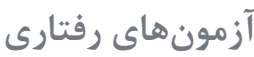

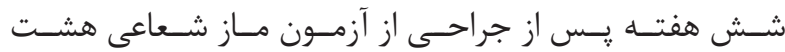

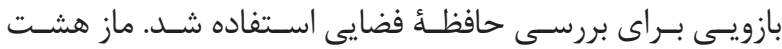

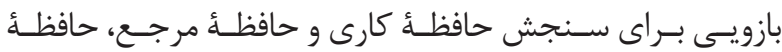

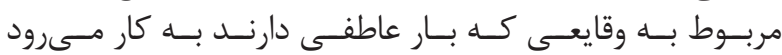

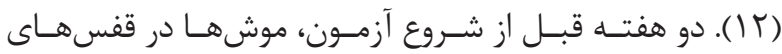

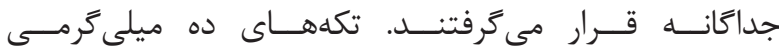

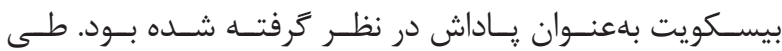

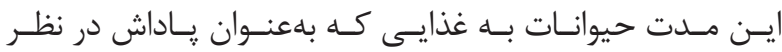

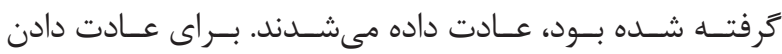

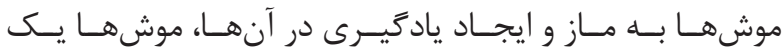

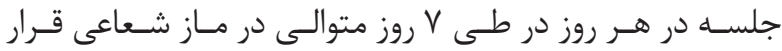

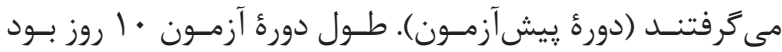

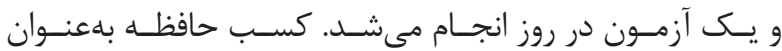

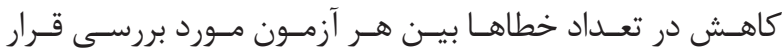

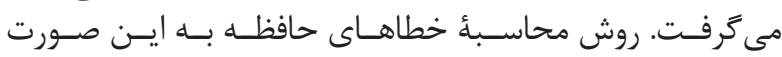

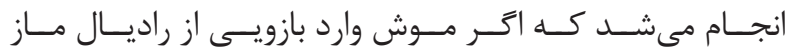

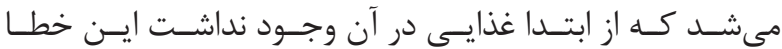

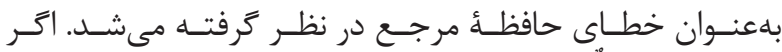

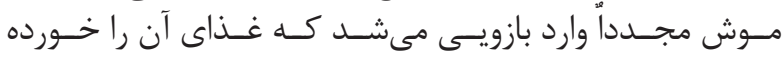

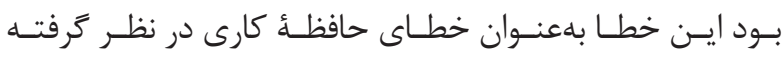

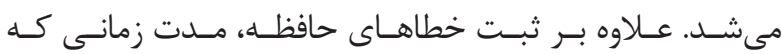

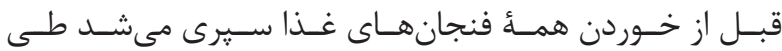

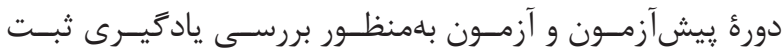
مى شــد. استرس ملايم مزمن غير قابل يبيشبينى

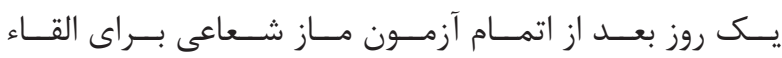

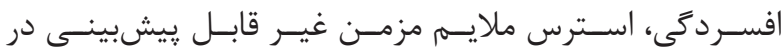

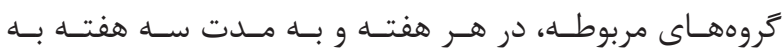

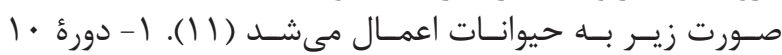

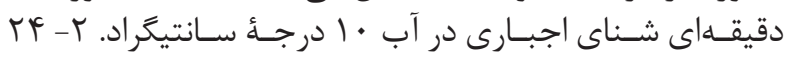

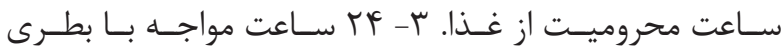

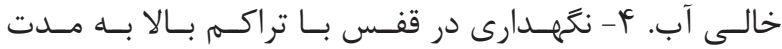
YF

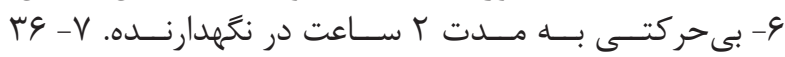

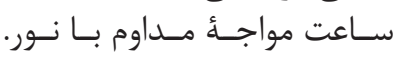

$$
\text { آزمون شناى اجبارى }
$$

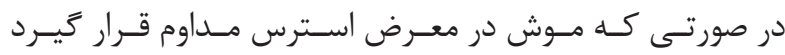

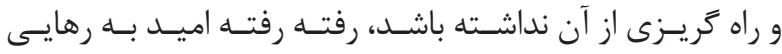

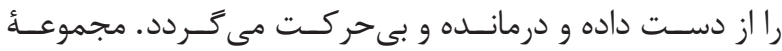

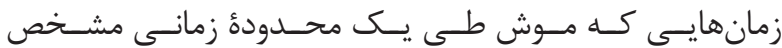

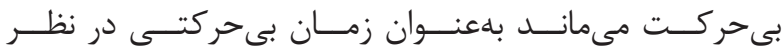

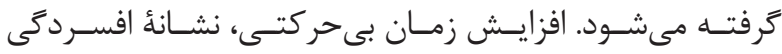

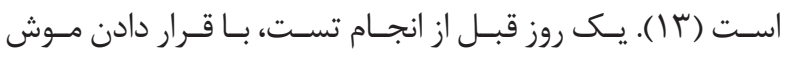

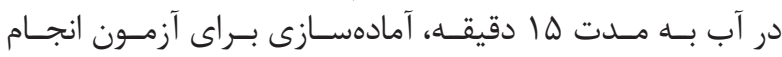

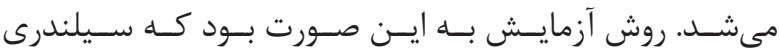




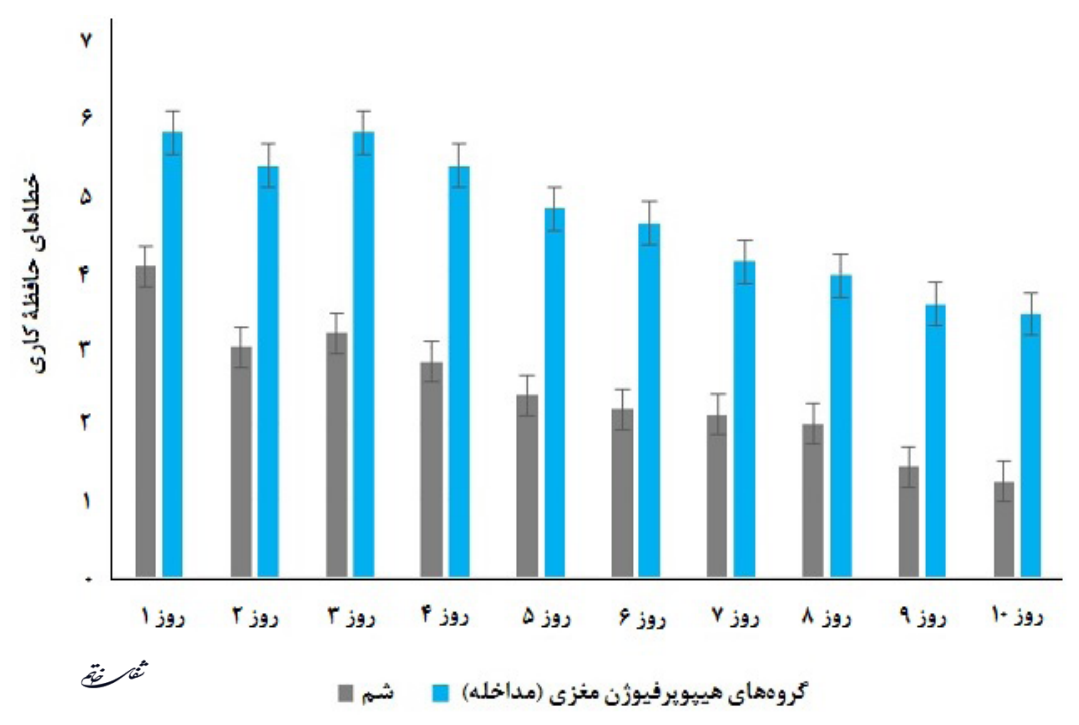

نمودار 1- تعداد خطاهاى حافظهٔ كارى در آزمون ماز شعاعى.

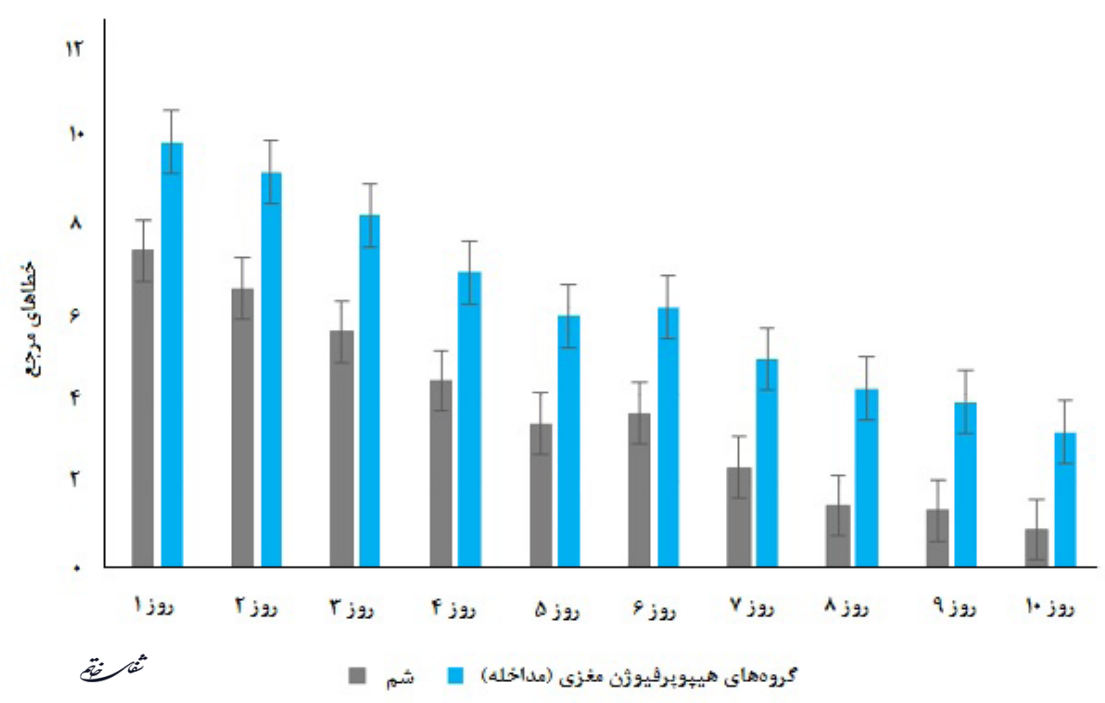

نمودار r- تعداد خطاهاى مرجع در آزمون ماز شعاعى.

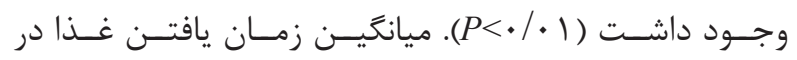

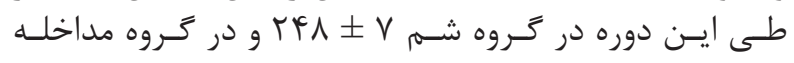

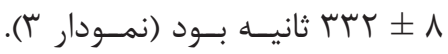

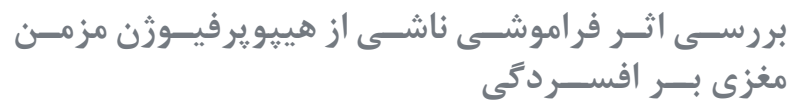
زمان بى حركتى

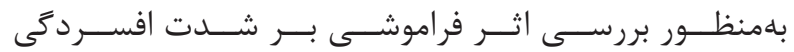

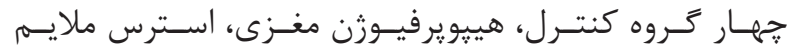

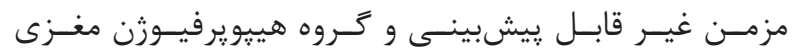

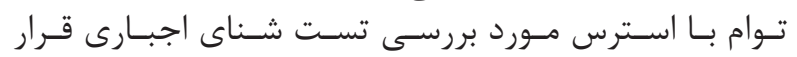

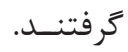

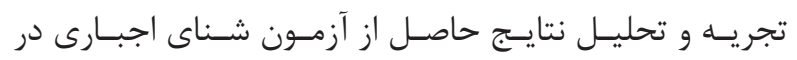

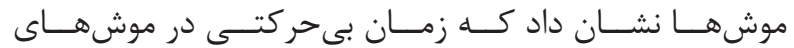

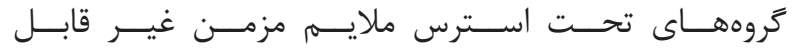

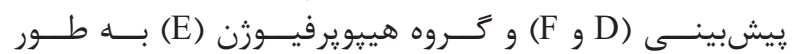

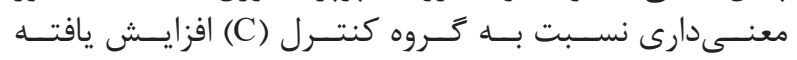

يادَيرى

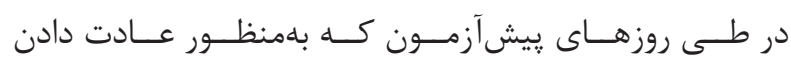

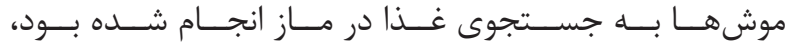

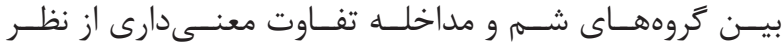

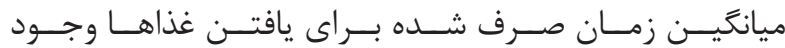

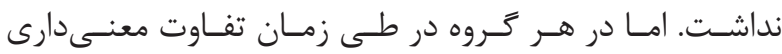

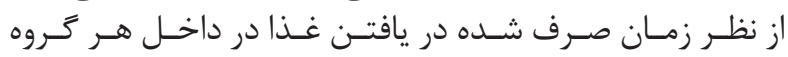

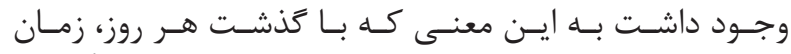

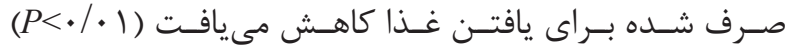

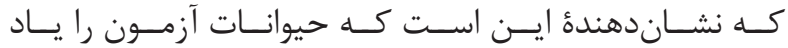

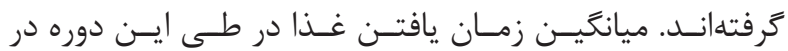
كَروه شـم ه

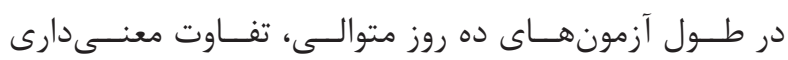

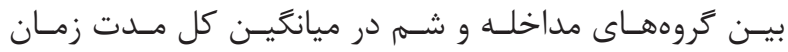

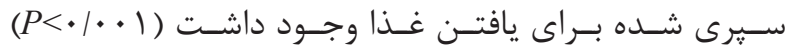

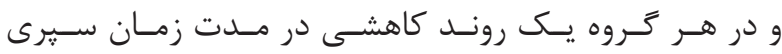

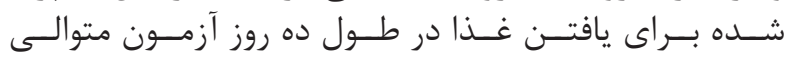




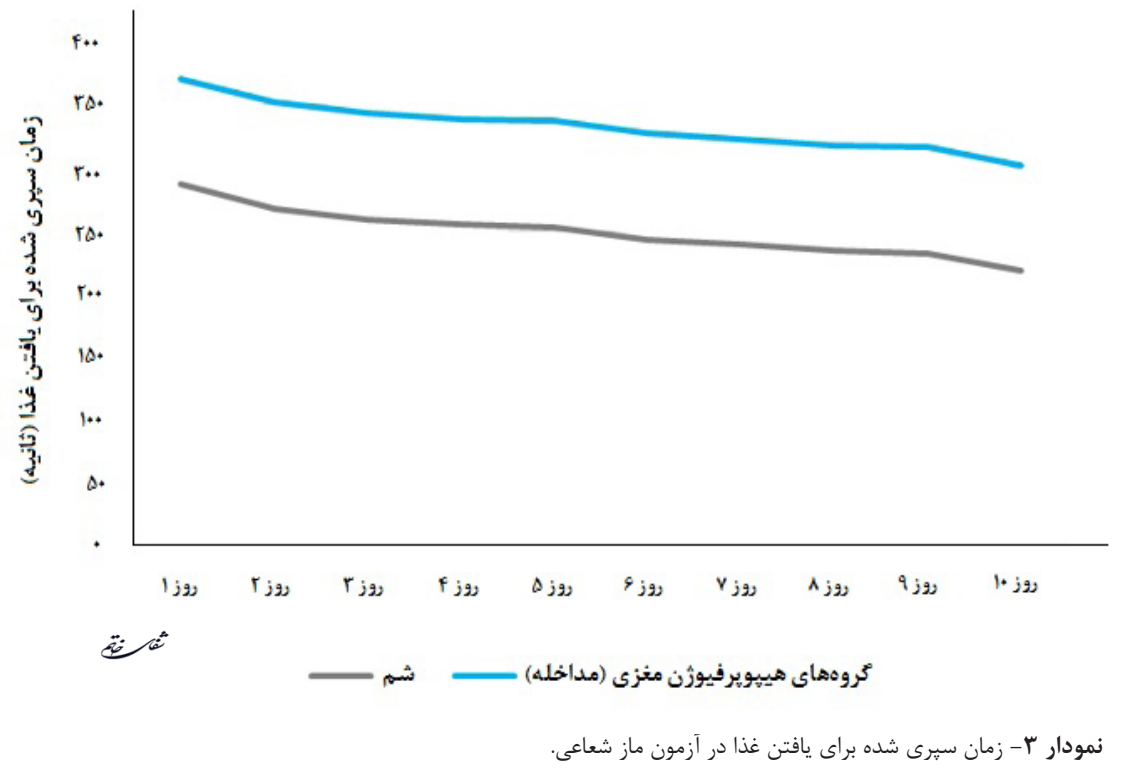

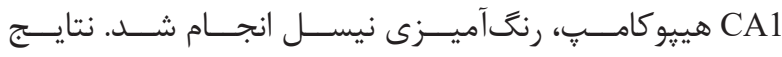

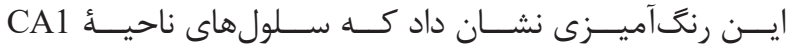

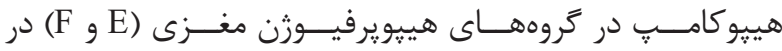

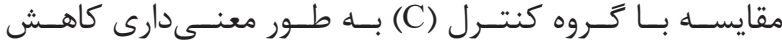

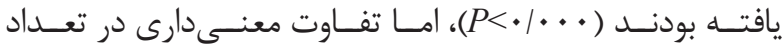

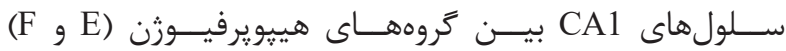

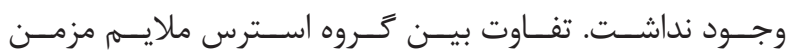

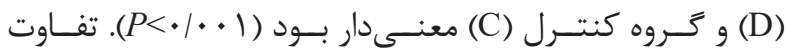

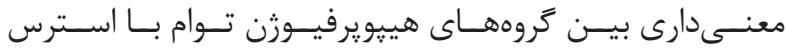

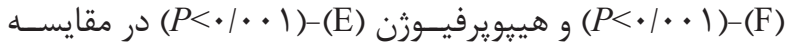

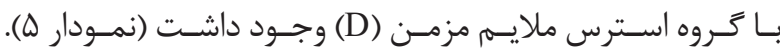

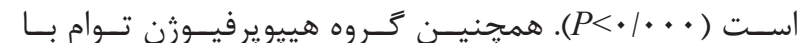

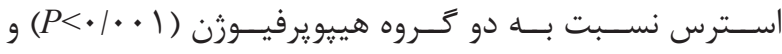

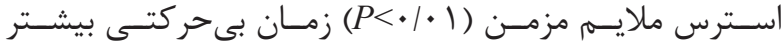

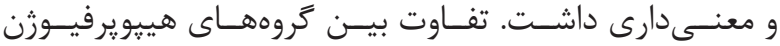

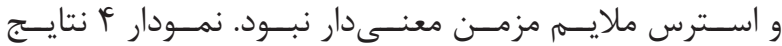

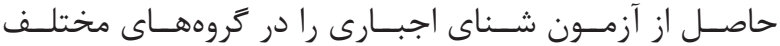

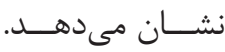

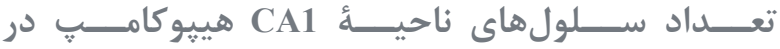

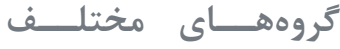
بهمنظـــور بررسـى و شــمارش تعــداد ســلول در ناحيــــ

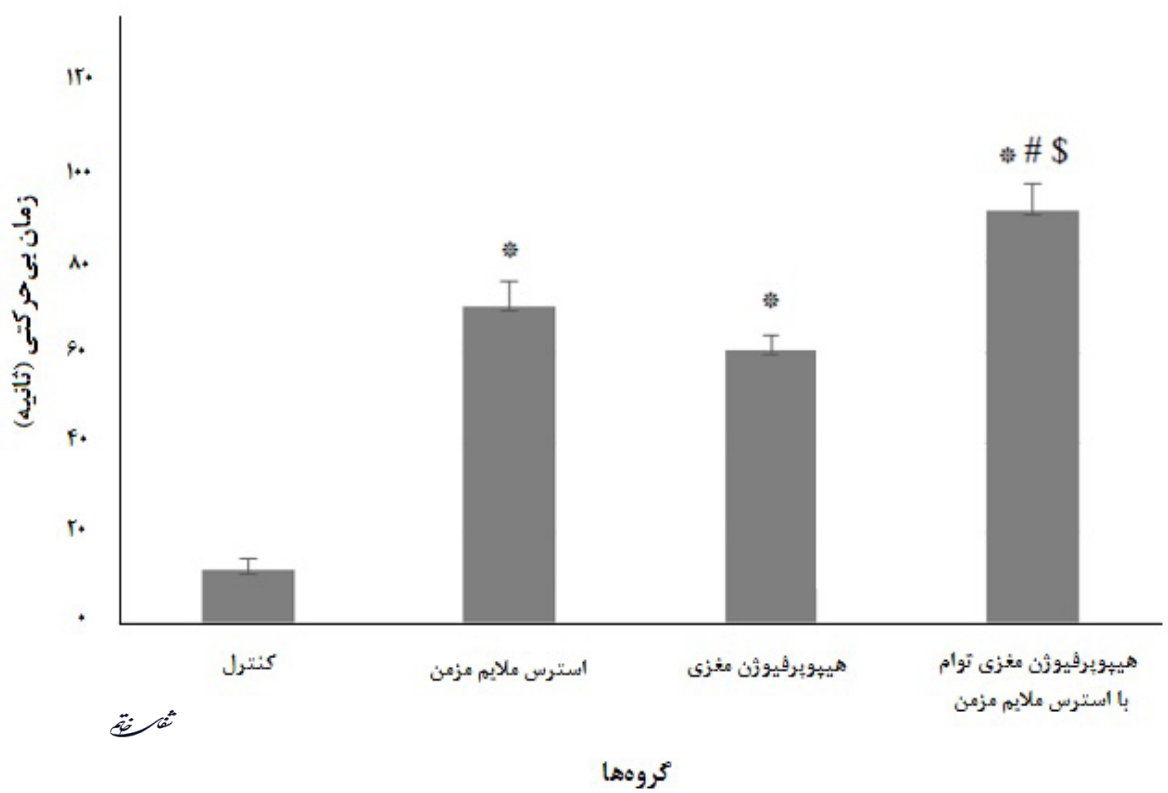

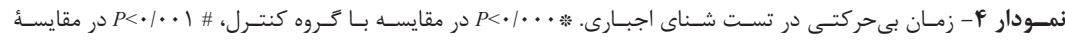

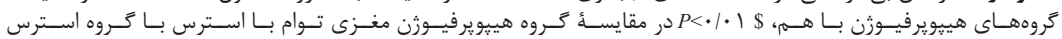



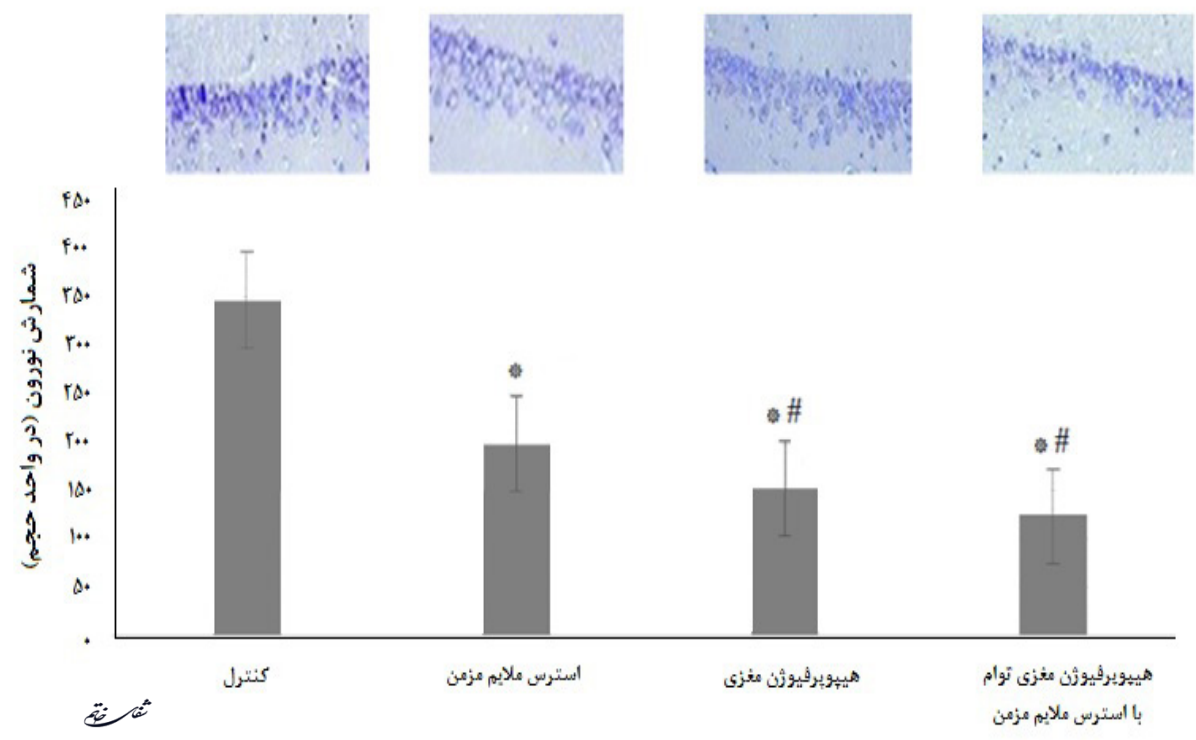

مروهها

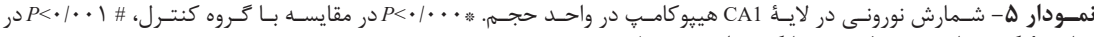

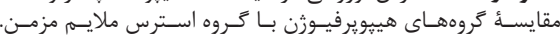

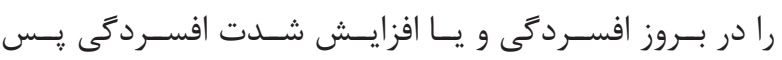

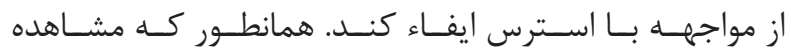

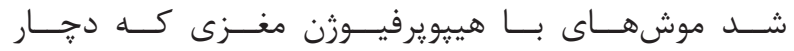

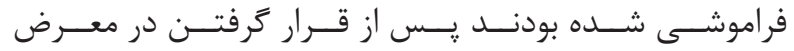

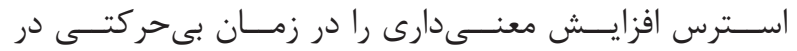

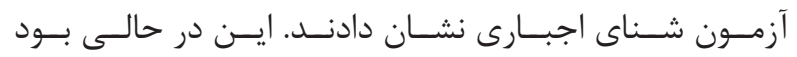

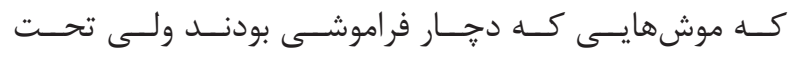

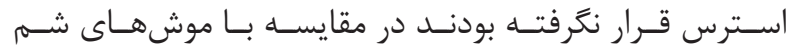

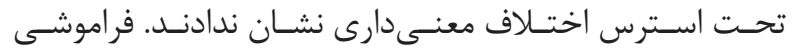

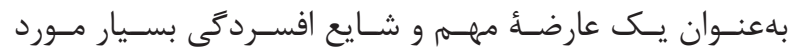

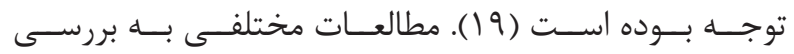

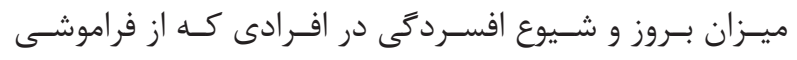

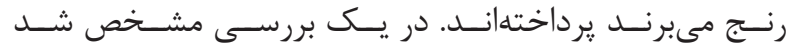

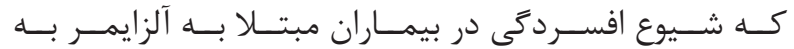

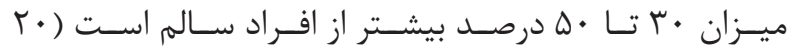
(V،

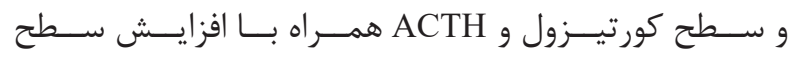

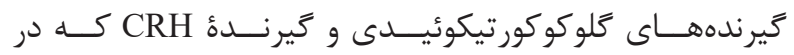

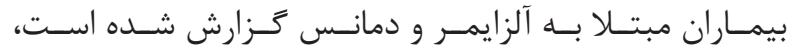

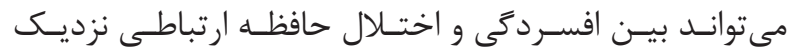

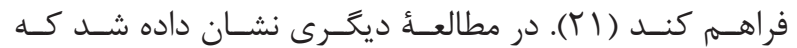

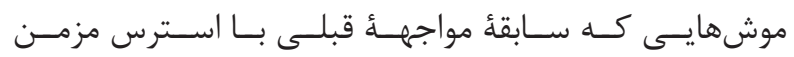

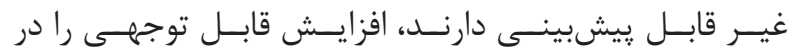

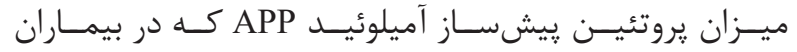

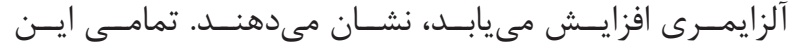

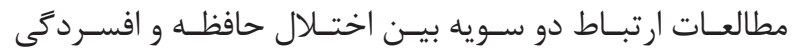

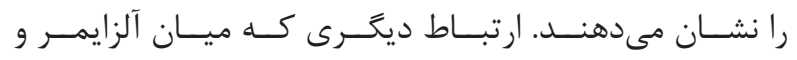

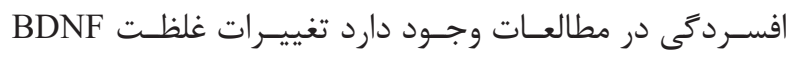

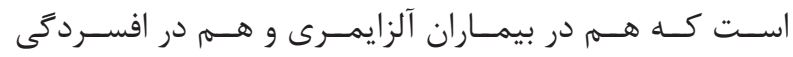

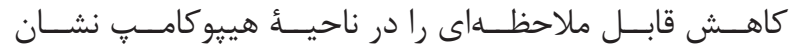

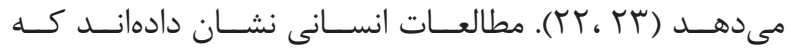

بحث و نتيجه كَيرى

بروز فراموشى در هيبويرفيوثن مزمن مغزى

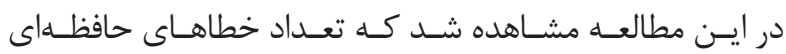

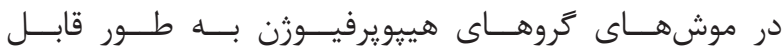

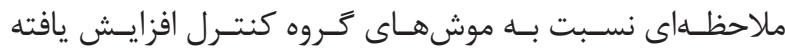

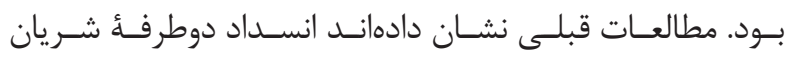

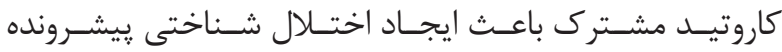

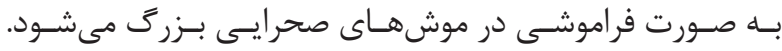

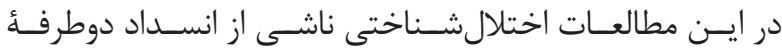

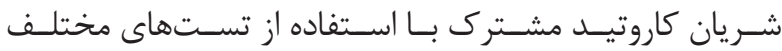

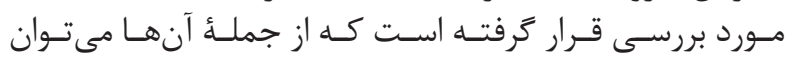

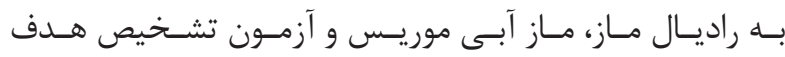

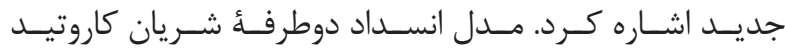

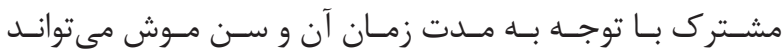

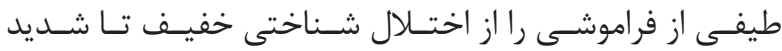

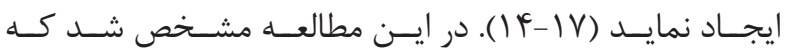

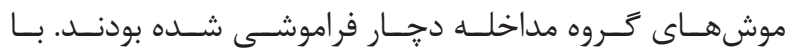

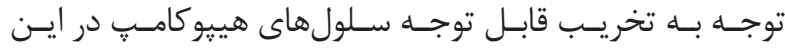

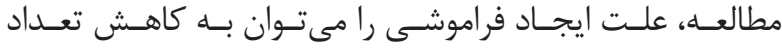

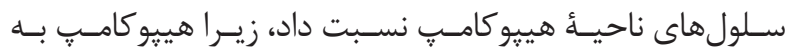

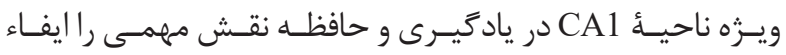
مى كنـــد (1) (1).

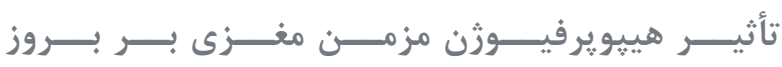

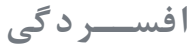

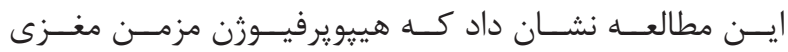

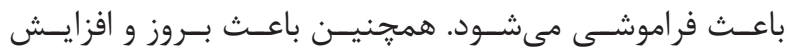

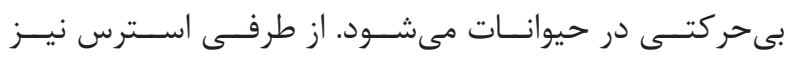

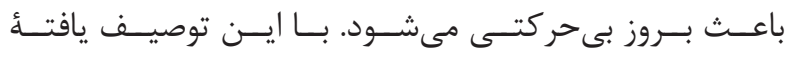

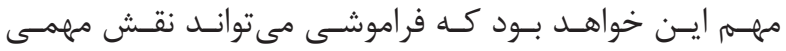




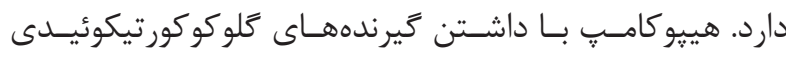

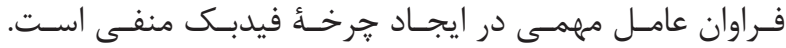

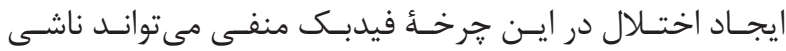

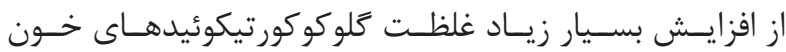

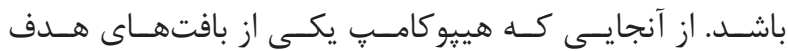

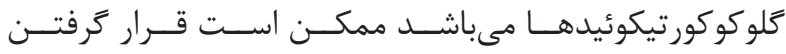

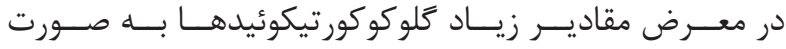

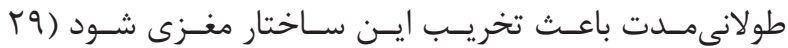

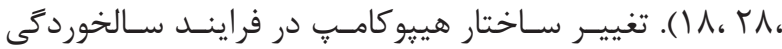

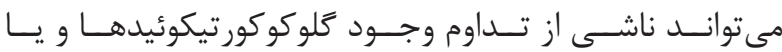

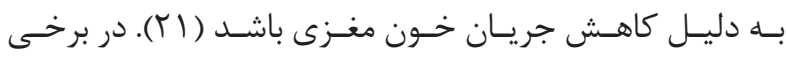

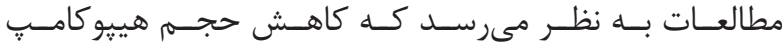

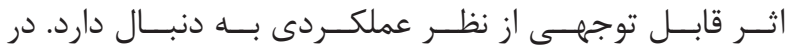

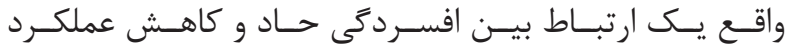

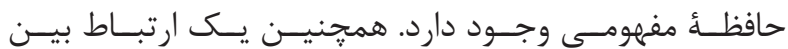

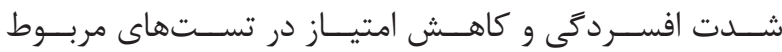

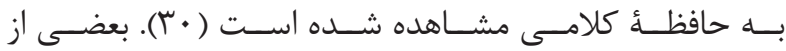

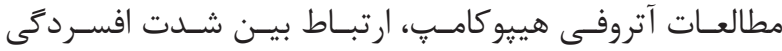

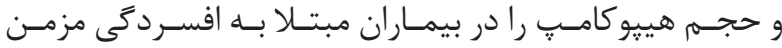

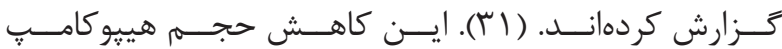

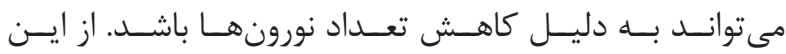

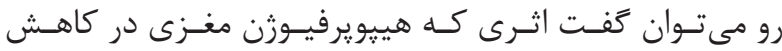

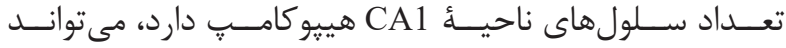

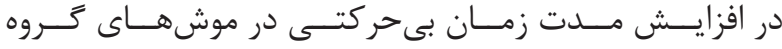

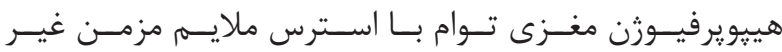

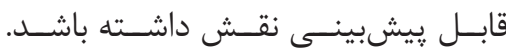

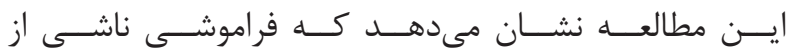

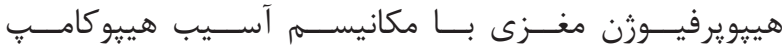

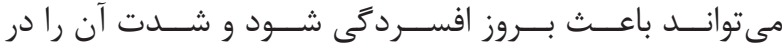

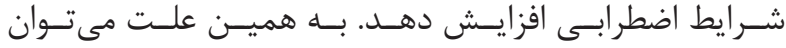

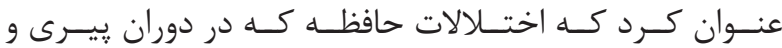

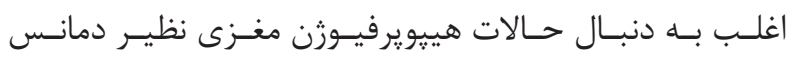

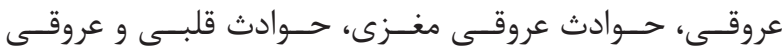

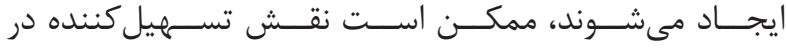

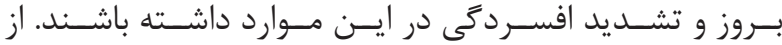

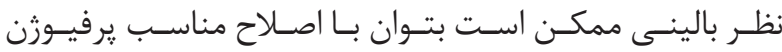

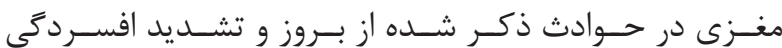

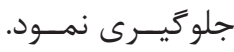

1. O'Brien JT, Thomas A. Vascular dementia. Lancet. 2015; 386(10004): 1698-706.

2. de la Torre JC. Are major dementias triggered by poor blood flow to the brain? theoretical considerations. J Alzheimers Dis. 2017; 57(2): 353-71.

3. Du SQ, Wang XR, Xiao LY, Tu JF, Zhu W, He T, et al. Molecular mechanisms of vascular dementia: what can be learned from animal models of chronic cerebral hypoperfusion? Mol Neurobiol. 2017; 54(5): 3670-82.

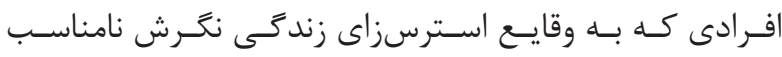

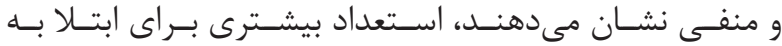

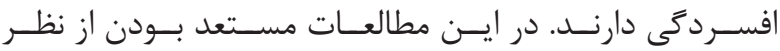

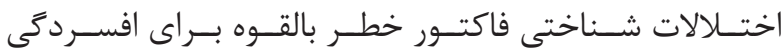

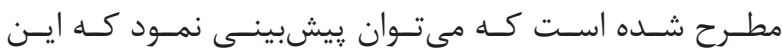

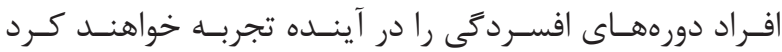

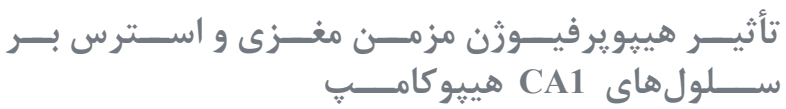

شـــمارش ســلولهاى ناحيـــ

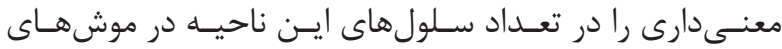

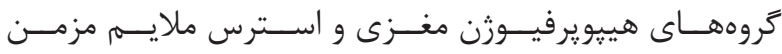

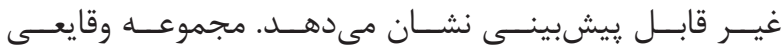

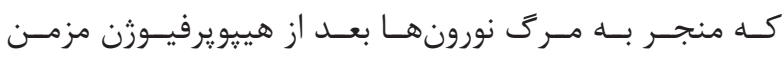

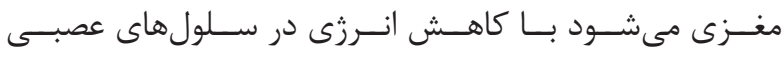

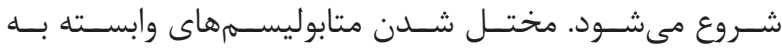

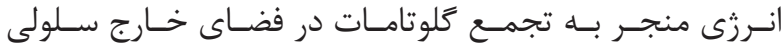

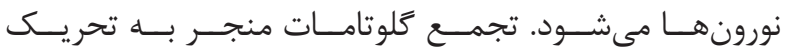

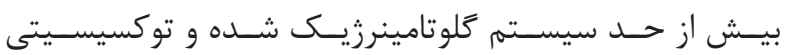

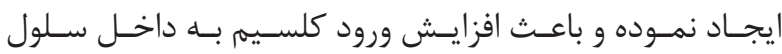

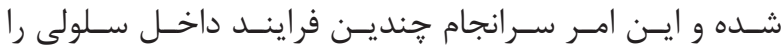

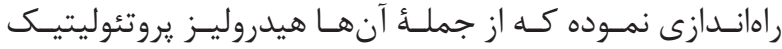

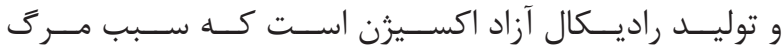

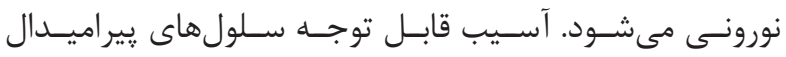

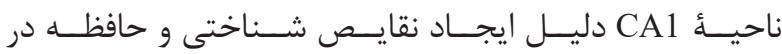

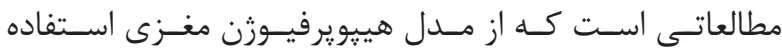

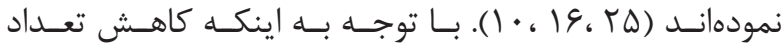

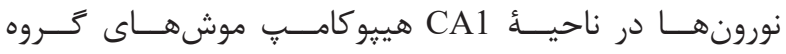

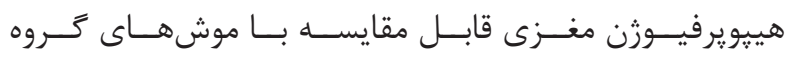

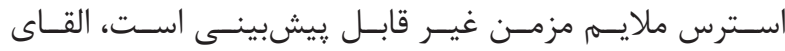

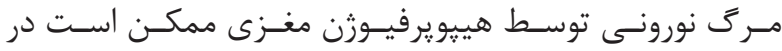

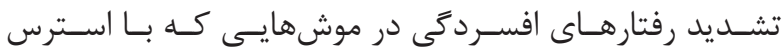

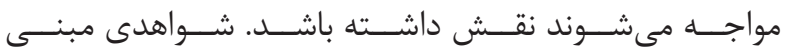

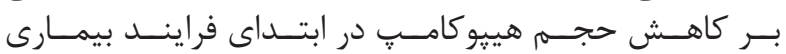

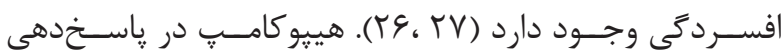

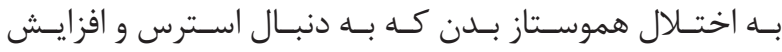

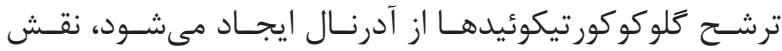

منابع

4. Rosenberg GA. Extracellular matrix inflammation in vascular cognitive impairment and dementia. Clin Sci (Lond). 2017; 131(6): 425-37.

5. Vijayan M, Reddy PH. Stroke, vascular dementia, and alzheimer's disease: molecular links. J Alzheimers Dis. 2016; 54(2): 427-43.

6. Behrman S, Valkanova V, Allan CL. Diagnosing and managing mild cognitive impairment. Practitioner. 2017; 261(1804): 17-20. 
7. Ismail Z, Elbayoumi H, Fischer CE, Hogan DB, Millikin CP, Schweizer T, et al. Prevalence of depression in patients with mild cognitive impairment: a systematic review and meta-analysis. JAMA Psychiatry. 2017; 74(1): 58-67.

8. Mourao RJ, Mansur G, Malloy-Diniz LF, Castro Costa E, Diniz BS. Depressive symptoms increase the risk of progression to dementia in subjects with mild cognitive impairment: systematic review and metaanalysis. Int J Geriatr Psychiatry. 2016; 31(8): 905-11.

9. Kastenschmidt EK, Kennedy GJ. Depression and anxiety in late life: diagnostic insights and therapeutic options. Mt Sinai J Med. 2011; 78(4): 527-45.

10. Patel A, Moalem A, Cheng H, Babadjouni RM, Patel $\mathrm{K}$, Hodis DM, et al. Chronic cerebral hypoperfusion induced by bilateral carotid artery stenosis causes selective recognition impairment in adult mice. Neurol Res. 2017; 39(10): 910-7.

11. Willner P. The chronic mild stress (CMS) model of depression: History, evaluation and usage. Neurobiol Stress. 2017; 6: 78-93.

12. Crystal JD. Animal models of source memory. J Exp Anal Behav. 2016; 105(1): 56-67.

13. Yankelevitch-Yahav R, Franko M, Huly A, Doron $\mathrm{R}$. The forced swim test as a model of depressive-like behavior. J Vis Exp. 2015; (97): doi: 10.3791/52587.

14. Choi JY, Cui Y, Kim BG. Interaction between hypertension and cerebral hypoperfusion in the development of cognitive dysfunction and white matter pathology in rats. Neuroscience. 2015; 303: 115-25.

15. Huang Y, Fan S, Li J, Wang YL. Bilateral common carotid artery occlusion in the rat as a model of retinal ischaemia. Neuro-ophthalmology. 2014; 38(4): 180-8.

16. Jing Z, Shi C, Zhu L, Xiang Y, Chen P, Xiong Z, et al. Chronic cerebral hypoperfusion induces vascular plasticity and hemodynamics but also neuronal degeneration and cognitive impairment. J Cereb Blood Flow Metab. 2015; 35(8): 1249-59.

17. Sarti C, Pantoni L, Bartolini L, Inzitari D. Cognitive impairment and chronic cerebral hypoperfusion: what can be learned from experimental models. J Neurol Sci. 2002; 203-204: 263-6.

18. Knierim JJ. The hippocampus. Curr Biol. 2015; 25(23): R1116-21.

19. Mirza SS, Ikram MA, Bos D, Mihaescu R, Hofman
A, Tiemeier H. Mild cognitive impairment and risk of depression and anxiety: A population-based study. Alzheimers Dement. 2017; 13(2): 130-9.

20. Roca M, Vives M, Lopez-Navarro E, Garcia-Campayo J, Gili M. Cognitive impairments and depression: a critical review. Actas Esp Psiquiatr. 2015; 43(5): 187-93.

21. Bartsch T, Wulff P. The hippocampus in aging and disease: From plasticity to vulnerability. Neuroscience. 2015; 309: 1-16.

22. Xie F, Zhao Y, Ma J, Gong JB, Wang SD, Zhang $\mathrm{L}$, et al. The involvement of homocysteine in stressinduced beta precursor protein processing and related cognitive decline in rats. Cell Stress Chaperones. 2016; 21(5): 915-26.

23. Tsai SJ. Brain-derived neurotrophic factor: a bridge between major depression and Alzheimer's disease? Med Hypotheses. 2003; 61(1): 110-3.

24. Alexopoulos GS. Depression in the elderly. Lancet. 2005; 365(9475): 1961-70.

25. Ihara M, Taguchi A, Maki T, Washida K, Tomimoto H. A mouse model of chronic cerebral hypoperfusion characterizing features of vascular cognitive impairment. Methods Mol Biol. 2014; 1135: 95-102.

26. Czeh B, Lucassen PJ. What causes the hippocampal volume decrease in depression? Are neurogenesis, glial changes and apoptosis implicated? Eur Arch Psychiatry Clin Neurosci. 2007; 257(5): 250-60.

27. Sierksma AS, Van den Hove DL, Steinbusch HW, Prickaerts J. Major depression, cognitive dysfunction and Alzheimer's disease: Is there a link? Eur J Pharmacol. 2010; 626(1): 72-82.

28. Kim BK, Ko IG, Kim SE, Kim CJ, Yoon JS, Baik $\mathrm{HH}$, et al. Impact of several types of stresses on shortterm memory and apoptosis in the hippocampus of rats. International Neurourology Journal. 2013; 17(3): 114-20.

29. Pawluski JL, Lambert KG, Kinsley CH. Neuroplasticity in the maternal hippocampus: Relation to cognition and effects of repeated stress. Horm Behav. 2016; 77: 86-97.

30. Muneer A, Mazommil R. The staging of major mood disorders: clinical and neurobiological correlates. Psychiatry Investigation. 2018; 15(8): 747-58.

31. Brown ES, Hughes CW, McColl R, Peshock R, King KS, Rush AJ. Association of depressive symptoms with hippocampal volume in 1936 adults. Neuropsychopharmacology. 2014; 39(3): 770-9. 